\title{
LOCALIZED OPTICAL EXCITATIONS AND TWO-EXCITON SPECTROSCOPY OF PHENYLACETYLENE DENDRIMERS
}

\author{
V. CHERNYAK, E.Y. POLIAKOV, S. TRETIAK, S. MUKAMEL \\ Department of Chemistry, University of Rochester, Rochester, New York 14627
}

\begin{abstract}
The one- and two-exciton manifolds of conjugated dendrimers possessing fractal geometries are studied using the Frenkel exciton model. Two-photon spectra can be used to determine both the magnitude and the sign of short-range coupling among segments. Self-similarity and the high degree of symmetry make it possible to compute the one-exciton states and the optical response with reduced numerical effort that scales linearly rather than exponentially with the number of generations. The third-order optical response and exciton scattering matrix are expressed in compact forms using irreducible representation of optical excitations, totally avoiding the expensive explicit calculation of two-exciton eigenstates.
\end{abstract}

\section{INTRODUCTION}

In this article we study the linear and nonlinear optical excitations of conjugated phenylacetylene dendrimers which form tree-like structures by connecting phenylacetylene oligomer segments at the meta-positions [1-3]. These supramolecules have been synthesized in a search for artificial antenna systems that mimic biological energy transfer processes [1, 4-8]. The first five members of the compact family which has the same linear unit in all generations and constitutes the subject of this study are shown in Fig. 1. Using the notation of Ref. [9], we denote the dendrimer with $l$ generations by $D l$.

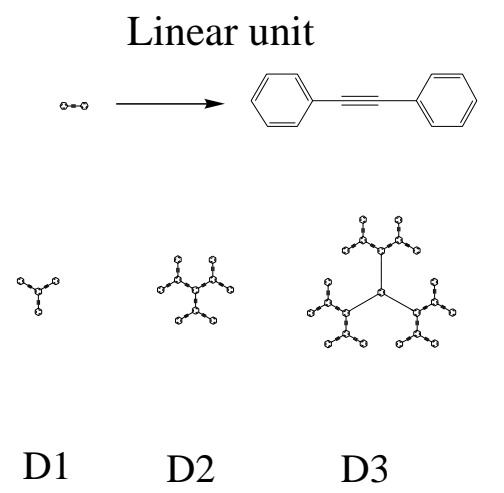

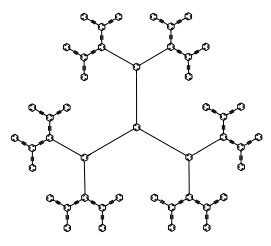

D4

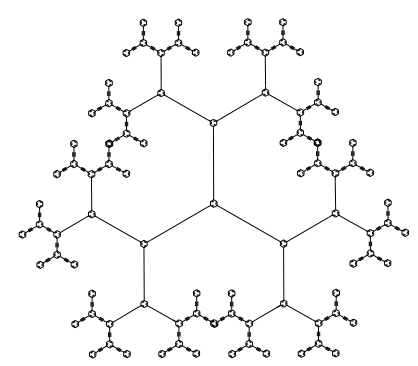

D5

Figure 1: Structures of the compact phenylacetylene dendrimers family made of the same linear building unit.

Self-similar geometry leads to unusual transport and optical properties in these Cayley tree or "Bethe lattice" structures [2-11]. Application of quantum chemistry methods to calculate the electronic structure of giant molecules is limited by computational power to small systems $[12,13]$. The theoretical investigation is complicated by the delocalized nature of electronic excitations, strong electron correlations, and vibronic coupling [12, 13]. The problem is simplified considerably when a molecule can be divided into a set of chromophores which are well separated in space, and their interactions are purely Coulombic. Electron 
exchange is then negligible, each chromophore retains its own electrons, and the system may be described using the Frenkel exciton Hamiltonian [14, 15].

It has been shown in [16] that optical excitations in these dendrimers do not involve charge separation between different segments. This implies that optical excitations are localized in the sense that the relative motion of electrons and holes is restricted to a single segment. However, the center of mass motion of electron-hole pairs can still be delocalized across the entire molecule. This localization allows to describe the optical response of the dendrimer using the Frenkel (rather than charge transfer) exciton Hamiltonian [14, 15, 17]:

$$
H=\sum_{\bar{n}} \Omega_{\bar{n}} B_{\bar{n}}^{\dagger} B_{\bar{n}}+\sum_{\bar{n} \neq \bar{m}} J_{\bar{n} \bar{m}} B_{\bar{m}}^{\dagger} B_{\bar{n}}
$$

Each segment is modeled as a two-level chromophore (the ground and excited states of a single acetylene chain) [9]. $B_{\bar{m}}\left(B_{\bar{m}}^{\dagger}\right)$ are the annihilation (creation) operator of an excitation localized on the $\bar{m}$-th chromophore. They satisfy the commutation relations, $\left[B_{\bar{n}}, B_{\bar{m}}^{\dagger}\right]=$ $\delta_{\bar{n} \bar{m}}\left(1-2 B_{\bar{m}}^{\dagger} B_{\bar{m}}\right)$ and $\left[B_{\bar{n}}, B_{\bar{m}}\right]=\left[B_{\bar{n}}^{\dagger}, B_{\bar{m}}^{\dagger}\right]=\left(B_{\bar{m}}^{\dagger}\right)^{2}=\left(B_{\bar{m}}\right)^{2}=0$. The parameters of the Frenkel exciton Hamiltonian [Eq. (1)] have been computed using electronic structure calculations for $D 1$ [16]. The Coulomb coupling between the chromophores described by $J_{\bar{m} \bar{n}}$ leads to delocalization of energy due to the center of mass motion of the Frenkel excitons [9].

The structure of Frenkel excitons is very different for the compact and extended families of dendrimers where the linear segment length varies from generation to generation, which leads to a substantial difference in optical properties. In extended dendrimers, the segment length increases towards the center. This implies that the transition frequencies $\Omega_{\bar{n}}$ of the effective chromophores are the same only within the same generation and decrease towards the center forming an energy funnel. Since the differences between the transition frequencies in different generations are in the range $800-3200 \mathrm{~cm}^{-1}$ whereas the Coulomb coupling $J \sim 70 \mathrm{~cm}^{-1}[9]$, the excitons in extended dendrimers can only be delocalized within a given generation. In compact dendrimers the linear units are identical and, therefore, the excitons can be delocalized over the entire molecule. This implies that high-resolution optical spectra (i.e. with resolution higher than $J$ ) are much richer in the compact family. This has been our motivation for studying the high-resolution linear absorption spectra of compact dendrimers [9]. In this manuscript we extend this study to nonlinear optical spectroscopy.

Direct computation of the linear response of dendrimers constitutes a complicated task even for the Frenkel exciton model since the number of chromophores $N$ scales exponentially with the number of generations $l$. Utilizing the high symmetry, the problem of the linear response has been reduced to the diagonalization of up to $l \times l$ matrices [9]. Utilizing the symmetry, the computation of two-exciton states involves the diagonalization of $l^{3} \times l^{3}$ matrices. In this paper we use an alternative approach and express the signal in terms of the frequency dependent exciton scattering matrix $\bar{\Gamma}_{\bar{m} \bar{n}}(\omega)[17,18]$. This reduces the problem to inverting $l \times l$ matrices. The paper is organized as follows. In the next section we give a brief survey of the Frenkel exciton model for dendrimers and the classification of its one-exciton states [9]. In the following section we compute the two-photon nonlinear spectra. In the last section we give a brief summary of the results. Details of the derivations are given in the Appendices.

\section{FRENKEL EXCITON MODEL AND ONE-EXCITON STATES IN COMPACT DENDRIMERS}

Each generation is formed by a collection of phenylacetylene units separated by a fixed number of meta-conjugations. We consider the linear segment as a chromophore and only 
retain the strongest Coulomb interactions represented by the nearest neighbor couplings, i.e. coupling between linear segments connected through a single meta-conjugation. The effective aggregate with the dual Bethe lattice geometry is shown in Fig. 2. The set of

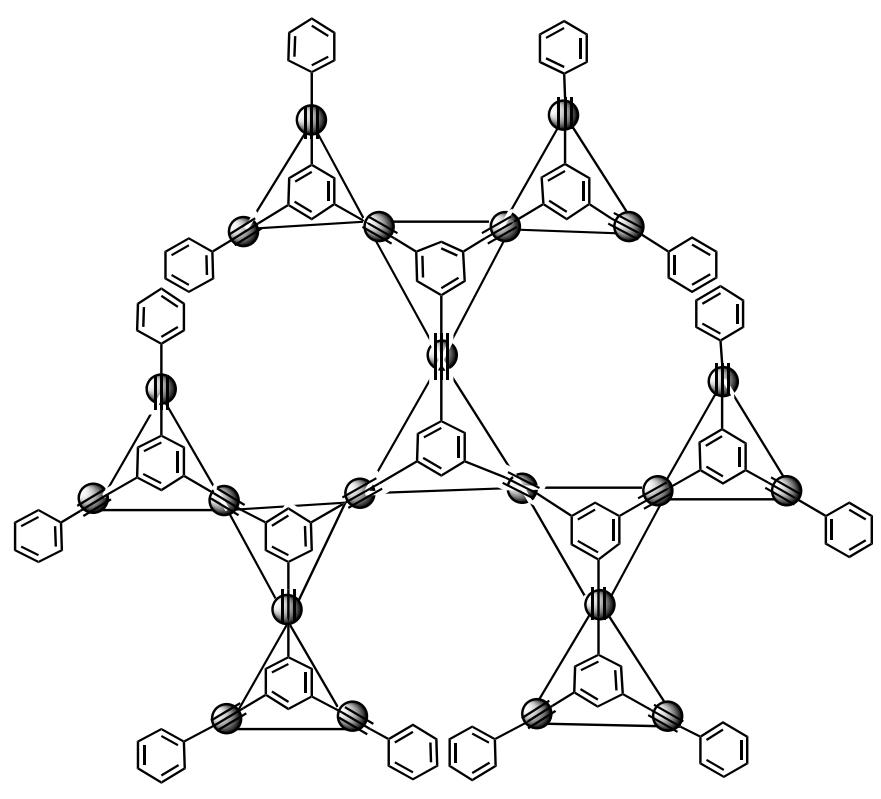

Figure 2: Dual Bethe lattice with triangular cycles of nearest-neighbor interactions.

chromophores is denoted by $X_{l}$, individual chromophores are labeled by Roman index with an overbar, $\bar{m}$, and one-exciton eigenstates by overrbared Greek indices, e.g. $\bar{\alpha}$. $\Omega_{\bar{n}}$ represents the electronic transition energy of the $\bar{n}$-th chromophore. All chromophores have the same transition frequency $\Omega_{\bar{n}}=\Omega$. Nearest neighbor coupling constants have the same magnitude $|J|=68 \mathrm{~cm}^{-1}$. Finding their signs is a delicate issue described in [9]. The number of chromophores in a generation scales with generation number $j$ as

$$
N_{j}=3 \cdot 2^{j-1}
$$

The total number of chromophores in $D l$ is $N=\sum_{j=1}^{l} N_{j}=3\left(2^{l}-1\right)$.

Below we survey the properties of one exciton states which also form the basis for computing the third-order nonlinear signal and the two-exciton states. The structure and dynamics of these states provides a sensitive probe for the coupling pattern and connectivity of dendrimers. Using the strong symmetry and decomposition of the entire one-exciton space discussed in Appendices $\mathrm{B}$ and $\mathrm{C}$ of [9], the excitons can be partitioned into two classes: antisymmetric and symmetric.

An antisymmetric exciton (Fig. 3, left panel) is characterized by its origin, the chromophore where the exciton starts, $\bar{m}$, and the energy quantum number, $\alpha$. For each $\bar{m} \in X_{l}$, $|\bar{m}|$ denotes the generation number to which $\bar{m}$ belongs. We note that $|\bar{m}| \neq l$, so that $\alpha=1,2, \ldots, l-|\bar{m}|$. The wavefunction of an exciton $\phi_{\bar{m} \alpha}$ which originates at $\bar{m}$ is represented in Fig. 3 where $j=l-|\bar{m}|$. All wavefunctions $\phi_{\bar{m} \alpha}(\bar{n})$ which originate in $\bar{m}$ are nonzero for $\bar{n}$ which belong to the branches which start at $\bar{m}$. As shown in the Figure, a wavefunction $\phi_{\bar{m} \alpha}$ is determined by $j=l-|\bar{m}|$ numbers $\phi_{\alpha}^{(j)}(1), \ldots, \phi_{\alpha}^{(j)}(j)$. The coefficients $\phi_{\alpha}^{(j)}(1), \ldots, \phi_{\alpha}^{(j)}(j)$ satisfy the Schrödinger equations for one-exciton states [Eq. (A1) in Ref. [9]]. 

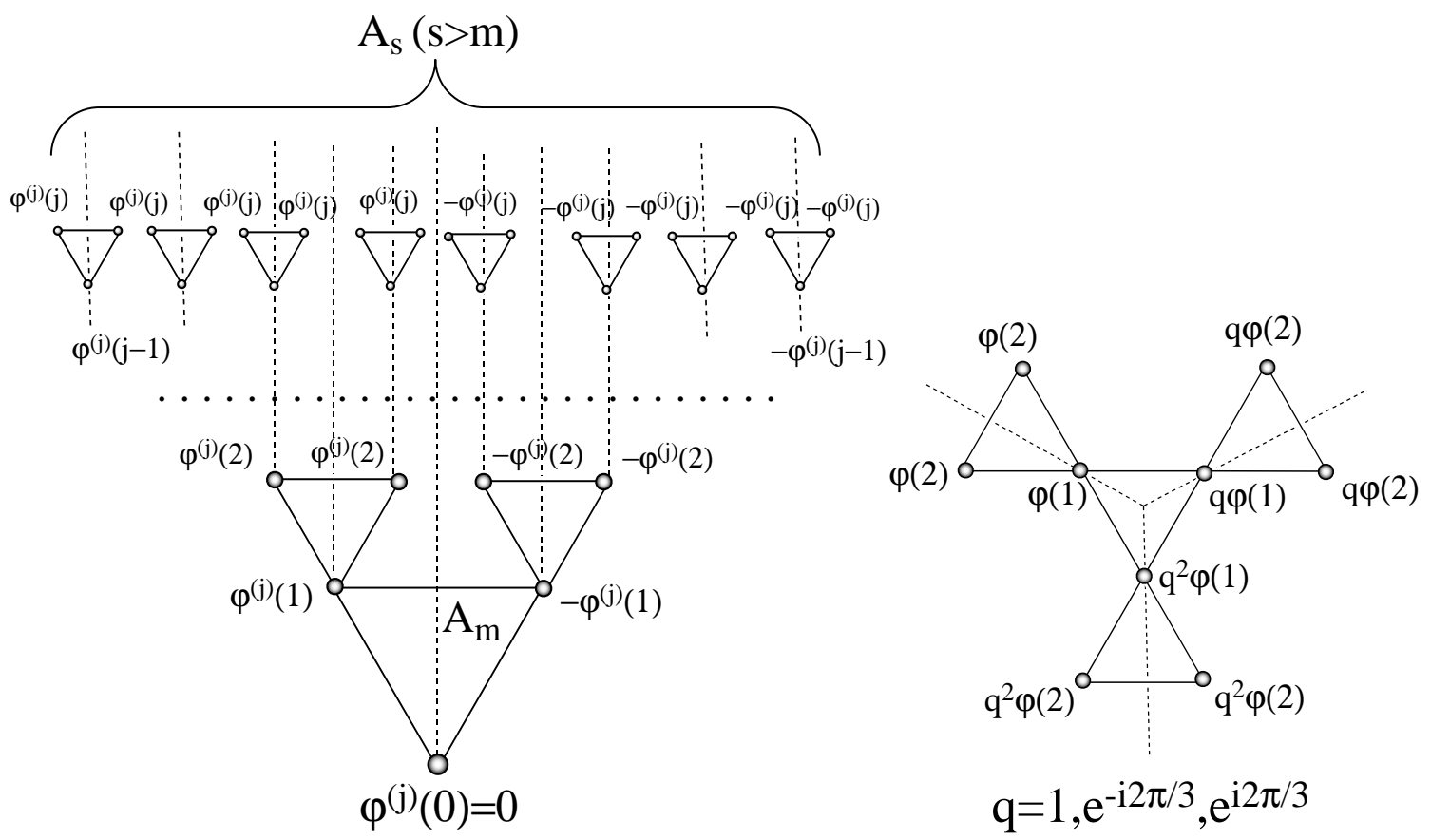

Figure 3: Left panel: The antisymmetric exciton of length $j$. The exciton wavefunction $\phi_{\alpha \bar{m}}$ is represented by $j=l-|\bar{m}|$ nonzero numbers $\phi^{(j)}(1), . ., \phi^{(j)}(j)$ (the superscript of exciton length $(j)$ and energy quantum number $\alpha$ are dropped). Right panel: Symmetric exciton and the representation of its wavefunction for the D3 molecule. (The subscript for $q_{t}$ and index $\alpha$ are dropped.)

The coefficients $\phi_{\alpha}^{(j)}(1), \ldots, \phi_{\alpha}^{(j)}(j)$ can be expressed in terms of the wavefunctions of an effective linear chain of length $j$ with nearest-neighbor hopping, $\psi_{\alpha}^{(j)}(i)$, [see Eq.(A6) in [9]]

$$
\phi_{\alpha}^{(j)}(i)=\psi_{\alpha}^{(j)}(i) / \sqrt{2^{i}} .
$$

We shall refer to excitons $\phi_{\bar{m} \alpha}$ with $|\bar{m}|=l-j$ as excitons of length $j$. Since an exciton of length $j$ with a given quantum number $\alpha(\alpha=1,2, \ldots j)$ can originate at any chromophore $\bar{m}$ with $|\bar{m}|=l-j$ we have altogether $3 \cdot 2^{l-j-1}$ excitons with energy $\epsilon_{\alpha}^{(j)}$. This corresponds to the number of chromophores in the $l-j$ generation [see Eq. (2)]. The total number of excitons of length $j$ is therefore $3 \cdot 2^{l-j-1} j$ where the last $j$ factor is related to the quantum number $\alpha$ and gives the number of distinct excitons of length $j$.

The symmetric excitons which have fixed length $l$ and consist of three branches are presented in the right panel of Fig. 3. Each exciton wavefunction is determined by the auxiliary wavefunctions $\tilde{\phi}_{\alpha}^{(t)}(1), \ldots, \tilde{\phi}_{\alpha}^{(t)}(j)$. These excitons are labeled by the quantum numbers

$$
q_{t}=e^{i 2 \pi(t-1) / 3}, \quad t=1,2,3,
$$

with energies $\epsilon_{\alpha, t}, t=1,2,3$, and $\alpha=1,2, \ldots, l$. For each $\alpha$, there are therefore three symmetric excitons, and the total number of excitons is $3 l$. The auxiliary wavefunctions $\tilde{\phi}_{\alpha}^{(t)}$ scale in the various branches as $\left(1, q_{t}, q_{t}^{2}\right)$. They can be also mapped onto the effective linear chain wavefunctions, $\tilde{\psi}_{\alpha}^{(t)}(i)$, [see Eqs. (A2) and (A6) in Ref. [9]]:

$$
\tilde{\phi}_{\alpha}^{(t)}(i)=\tilde{\psi}_{\alpha}^{(t)}(i) / \sqrt{3 \cdot 2^{i-1}} .
$$


Eqs. (3) and (5) map the one-exciton states in a compact dendrimer onto one-exciton states of effective linear chains with nearest neighbor coupling.

\section{THE FOUR-WAVE MIXING SIGNAL: THIRD ORDER SUSCEPTIBILITY AND EXCITON SCATTERING}

Frequency-domain four-wave mixing signal is described by the third-order susceptibility $\hat{\chi}^{(3)}[17,18]$ :

$$
\begin{aligned}
& \hat{\chi}^{(3)}\left(-\omega_{s} ; \omega_{1}, \omega_{2}, \omega_{3}\right)=\frac{1}{6} \sum_{\text {perm }} \sum_{\bar{m} \bar{i} \bar{j} \bar{k}} \mu_{\bar{m}} \mu_{\bar{i}} \mu_{\bar{j}} \mu_{\bar{k}} \times \\
& \sum_{\bar{n} \bar{l}} \mathrm{G}_{\bar{m} \bar{n}}\left(\omega_{s}\right) \mathrm{G}_{\overline{l i}}\left(\omega_{1}\right) \mathrm{G}_{\overline{l j}}\left(\omega_{2}\right) \mathrm{G}_{\bar{k} \bar{n}}^{\dagger}\left(-\omega_{3}\right) \bar{\Gamma}_{\bar{n} \bar{l}}\left(\omega_{1}+\omega_{2}\right)+\text { c.c. },
\end{aligned}
$$

where perm denotes the six permutations of pairs $\left(\mu_{i}, \omega_{i}\right)$ for $i=1-3$, c.c. stands for complex conjugation, and $\mu_{\bar{m}}$ denotes the transition dipole of the $m$-th chromophore. $G$ denotes the one-exciton Green function

$$
\mathrm{G}_{\bar{m} \bar{n}}(\omega)=\sum_{\bar{\alpha}} \frac{\phi_{\bar{\alpha}}(\bar{m}) \phi_{\bar{\alpha}}^{*}(\bar{n})}{\omega-\epsilon_{\bar{\alpha}}+i \gamma}
$$

where $\gamma$ is the exciton dephasing rate.

The two-exciton information is contained in the two-exciton scattering matrix, $\bar{\Gamma}_{\bar{m} \bar{n}}$, which has the form:

$$
\bar{\Gamma}_{\bar{m} \bar{n}}(\omega)=-2\left[F(\omega)^{-1}\right]_{\bar{m} \bar{n}} .
$$

Here $F(\omega)$ is the two-exciton Green function in a reference system where the excitons are represented by non-interacting bosons. It is given by the convolution of two one-exciton Green's functions [17, 20]:

$$
F_{\bar{m} \bar{n}}(\omega)=\int_{-\infty}^{\infty} \frac{d \omega^{\prime}}{2 \pi i} G_{\bar{m} \bar{n}}\left(\omega^{\prime}\right) G_{\bar{m} \bar{n}}\left(\omega-\omega^{\prime}\right)
$$

Upon the substitution of Eq. (7) into Eq. (9), the matrix elements $F_{\bar{m} \bar{n}}$ are expressed in terms of one-exciton wavefunctions:

$$
\mathrm{F}_{\bar{m} \bar{n}}(\omega)=\sum_{\bar{\alpha}, \bar{\beta}} \frac{\phi_{\bar{\alpha}}(\bar{m}) \phi_{\bar{\beta}}(\bar{m}) \phi_{\bar{\alpha}}^{*}(\bar{n}) \phi_{\bar{\beta}}^{*}(\bar{n})}{\omega-\epsilon_{\bar{\alpha}}-\epsilon_{\bar{\beta}}+2 i \gamma} .
$$

We consider a two-color heterodyne detected four-wave mixing signal generated by three incoming beams in the direction $\mathbf{k}_{s}=\mathbf{k}_{1}+\mathbf{k}_{2}-\mathbf{k}_{3}$ with $\omega_{3}=\omega_{1}$. The signal is then given by $\operatorname{Im} \hat{\chi}^{(3)}\left(-\omega_{2} ; \omega_{1}, \omega_{2},-\omega_{1}\right)$. Making the rotating wave approximation, we obtain

$$
\begin{array}{r}
S\left(\omega_{1}, \omega_{2}\right) \propto \operatorname{Im} \sum_{\bar{m} \bar{i} \bar{j} \bar{k}}\left(\mu_{\bar{m}} \cdot \hat{e}_{s}\right)\left(\mu_{\bar{i}} \cdot \hat{e}_{1}\right)\left(\mu_{\bar{j}} \cdot \hat{e}_{2}\right)\left(\mu_{\bar{k}} \cdot \hat{e}_{3}\right) \times \\
\sum_{\bar{n} \bar{l}} \mathrm{G}_{\bar{m} \bar{n}}\left(\omega_{2}\right) \mathrm{G}_{\bar{l} \bar{i}}\left(\omega_{1}\right) \mathrm{G}_{\overline{l j}}\left(\omega_{2}\right) \mathrm{G}_{\bar{k} \bar{n}}^{\dagger}\left(\omega_{1}\right) \bar{\Gamma}_{\bar{n} \bar{l}}\left(\omega_{1}+\omega_{2}\right) .
\end{array}
$$

where $\hat{e}_{1}, \hat{e}_{2}, \hat{e}_{3}$, and $\hat{e}_{s}$ represent the unit vectors in the directions of the polarization of the three incoming and heterodyne fields, respectively. 
In order to focus on the two-exciton states and avoid any interference with one-exciton states, we tune both frequencies $\omega_{1}$ and $\omega_{2}$ to be off-resonant with respect to the one-exciton manifold, keeping their sum $\omega_{1}+\omega_{2}$ resonant with the two-exciton states. This yields

$$
S\left(\omega_{1}, \omega_{2}\right) \propto \sum_{\bar{m}, \bar{n}}\left(\mu_{\bar{m}} \cdot \hat{e}_{s}\right)\left(\mu_{\bar{m}} \cdot \hat{e}_{3}\right)\left(\mu_{\bar{n}} \cdot \hat{e}_{1}\right)\left(\mu_{\bar{n}} \cdot \hat{e}_{2}\right) \operatorname{Im} \bar{\Gamma}_{\bar{m} \bar{n}}\left(\omega_{1}+\omega_{2}\right)
$$

For the particular choice of the polarizations: $\hat{e}_{s}=\hat{e}_{2}, \hat{e}_{1}=\hat{e}_{3}$, Eq. (12) yields the twocolor pump-probe signal. Eq. (12) represents the signal as a convolution of the scattering matrix $\bar{\Gamma}_{\bar{m} \bar{n}}$ which represents the internal geometry of the molecule, e.g. the connectivity, the Coulomb coupling strength and the factor $M_{\bar{m} \bar{n}}=\sum_{\bar{m}, \bar{n}}\left(\mu_{\bar{m}} \cdot \hat{e}_{s}\right)\left(\mu_{\bar{m}} \cdot \hat{e}_{3}\right)\left(\mu_{\bar{n}} \cdot \hat{e}_{1}\right)\left(\mu_{\bar{n}} \cdot \hat{e}_{2}\right)$ which is related to molecular geometry, i.e. its three-dimensional shape which involves the relative dipole orientations becomes extremely complicated as the number of generations is increased. The signal's dependence on geometry can be eliminated by choosing $\hat{e}_{s}=\hat{e}_{3}$, $\hat{e}_{1}=\hat{e}_{2}$, and averaging over all possible orientations of $\hat{e}_{3}$ and $\hat{e}_{2}$ which yields

$$
S \propto \mu^{4} \operatorname{Im} \sum_{\bar{m}, \bar{n}} \bar{\Gamma}_{\bar{m} \bar{n}}\left(\omega_{1}+\omega_{2}\right)
$$

where $\mu$ is the chromophore transition dipole.

In appendix A, we express the two-exciton scattering matrix $\bar{\Gamma}_{\bar{m} \bar{n}}$ in terms of one-exciton wavefunctions. We have decomposed the one-exciton space of states $W_{l}$ into the sum of irreducible representations of the dendrimer group of symmetry $G_{l}$ [9]. (Its calculation only requires inverting $l \times l$ matrices.)

Setting $|\mu|=1$, the third-order nonlinear signal, $S\left(\omega_{1}, \omega_{2}\right)$, is plotted in Fig. 4 as a function of the sum-frequency detuning from two-exciton resonance, $\Delta \omega=\omega_{1}+\omega_{2}-2 \Omega$ with $\gamma=8 \mathrm{~cm}^{-1}$.

These spectra consist of several well-separated resonances, and the number of peaks grows for higher generations. The spectrum of a given generation contains all the features of lower generations, which are shifted as the number of generations $l$ is increased. This can be rationalized using the following arguments. The structure of single-exciton states described above, namely the property that an exciton starts at a certain chromophore and spreads towards the boundary implies that the antisymmetric excitons which play the major role in optical signals do not change as the number of generations is increased. Consequently, new features are added to the linear absorption spectra as $l$ is increased whereas previous features remain unchanged [9]. The situation with two-exciton states is different since excitons of different lengths interact by means of Pauli exclusion. The resulting spectral shifts are small since the interaction is weak. The spectra also broaden with $l$. This can be rationalized using the variational principle since the space of two-exciton states of $D l$ can be considered as an extension of that of $D(l-1)$.

Increasing the number of generations further leads to the eventual saturation of the nonlinear signal: The spectra of $D 5$ and $D 6$ and higher $D l$ (not shown) are very similar. Saturation of the two-photon spectrum is the consequence of self-similarity, as was previously demonstrated for linear absorption [9]. We further note that the spectrum is asymmetric. The highest peaks are blue-shifted $(\Delta \omega>0)$ as in $J$-aggregates [19]. This can be used to determine the sign of $J$. The two-exciton band extends from $-7|J|$ to $3.5|J|$ with bandwidth $10.5|J| \approx 700 \mathrm{~cm}^{-1}$. This estimate can be used to evaluate the magnitude of the coupling constant. For infinite dendrimers, the one-exciton band extends from $-J(1+2 \sqrt{2})$ to $J(2 \sqrt{2}-1)$, giving a bandwidth $\sim 5.8|J|$. The two-exciton bandwidth, $10.5|J|$, is narrower 


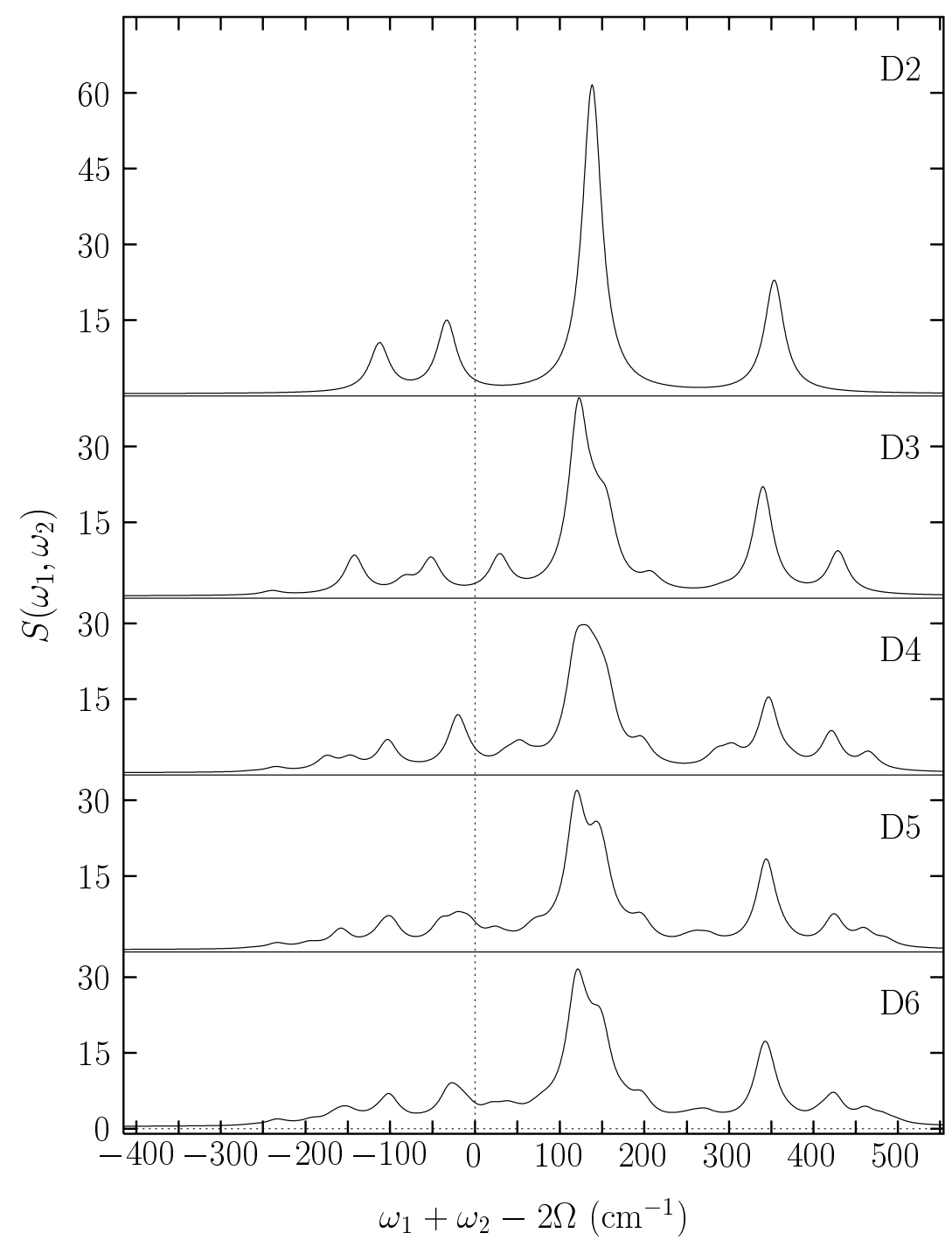

Figure 4: Four-wave mixing response signal from $D 2-D 6$ as a function of sum pump and signal frequency detuning from two-exciton resonance.

than twice the one-exciton bandwidth, 11.6 $J \mid$. This can be rationalized using the variational principle. The space of two-exciton states in the model of coupled two-level chromophores can be considered as a subspace of two-particle states in a system of non-interacting bosons obtained by excluding the double excitations on single chromophores. According to the variational principle, the two-exciton band is narrower than the two-boson band, which is precisely twice the one-exciton bandwidth.

\section{SUMMARY}

We have studied the two-color four-wave mixing signal in compact conjugated phenylacetylene dendrimers using the Frenkel exciton model. This model which usually applied for molecular aggregates may be used to describe optical excitations in dendrimers due to the localization of the relative motion of electrons and holes on the linear segments. This implies that as far as their optical properties are concerned, dendrimers behave like molecular 
aggregates rather than supermolecules. We have demonstrated that after a proper averaging over the orientations of the beam polarizations in a two-color four-wave mixing experiment, one obtains a signal which does not depend on the molecular three-dimensional geometry; The signal only reflects the connectivity and the Coulomb coupling. The scattering matrix approach to optical nonlinearities in Frenkel exciton systems [17, 18, 20] combined with utilization of the high symmetry turns out to be extremely powerful for computing nonlinear optical signals in dendrimers by considerably reducing the numerical effort. The effective size grows linearly rather than exponentially with $l$.

\section{ACKNOWLEDGMENTS}

This research was supported by the National Science Foundation and by the Air Force Office of Scientific Research. We wish to thank Prof. R. Kopelman and Prof. Y. Klafter for useful discussions.

\section{APPENDIX A: THE TWO-EXCITON SCATTERING MATRIX IN THE UNIT REPRESENTATION SUBSPACE}

In this Appendix we show how the high symmetry of a dendrimer can be used to compute the exciton scattering matrix $\bar{\Gamma}_{\bar{m} \bar{n}}(\omega)$. The most time consuming part of the computation is inverting the matrix $F_{\bar{m} \bar{n}}(\omega)$ which represents an operator $F(\omega)$ acting on the one-exciton space $W_{l}$ with dimension $N=3\left(2^{l}-1\right)$. The problem can be substantially simplified by noting that $F(\omega)$ is obviously a symmetric operator with respect to symmetry group $G_{l}$. Decomposing the space $W_{l}$ into a direct sum of irreducible representations (this has been carried out in [9]), $W_{l}=\oplus_{n=1}^{l-1} n V_{n} \oplus l \tilde{V}_{1} \oplus l \tilde{V}_{2} \oplus l \tilde{V}_{3}$, we can apply the Schur lemma to $F(\omega)$ which states that $F(\omega)$ does not have matrix elements between different irreducible representations whereas it can be described by an effective $n \times n$ matrix in the space $n V_{n}$ and by $l \times l$ matrices in the spaces $l \tilde{V}_{1}, l \tilde{V}_{2}$, and $l \tilde{V}_{3}$ (see [9] for the details). The operator $F(\omega)$ should therefore be projected into the spaces of irreducible representations and the corresponding $n \times n$ and $l \times l$ matrices should be inverted which yields the scattering operator $\bar{\Gamma}(\omega)$. In the case of the signal given by Eq. (13) it is easy to show that only the component of $\bar{\Gamma}(\omega)$ which acts in $l \tilde{V}_{1}$ is involved. $l \tilde{V}_{1}$ constitutes $l$ replicas of the one-dimensional unit representation. They are represented by $l$ vectors $v_{i}, i=1, \ldots, l$ in $W_{l}$ with components $v_{i}^{(\bar{m})}$ given by

$$
v_{i}^{(\bar{m})}=\frac{1}{\sqrt{N_{i}}} \delta_{|\bar{m}|, i}
$$

where $N_{i}$ is the number of chromophores in the $i$-th generation given by Eq. (2).

The matrix components of $F$ in the original basis and in the basis set $v_{i}$ are naturally related by

$$
F_{i j}=\sum_{\bar{m}, \bar{n}} v_{i}^{(\bar{m})} F_{\bar{m} \bar{n}} v_{j}^{(\bar{n}) *} .
$$

Upon substituting Eq. (10) into Eq. (15) we obtain that

$$
F_{i j}(\omega)=\sum_{\bar{m}, \bar{n}} \sum_{\bar{\alpha}, \bar{\beta}} \frac{v_{i}^{(\bar{m})} \phi_{\bar{\alpha}}(\bar{m}) \phi_{\bar{\beta}}(\bar{m})\left[\phi_{\bar{\alpha}}(\bar{n}) \phi_{\bar{\beta}}(\bar{n}) v_{j}^{(\bar{n})}\right]^{*}}{\omega-\epsilon_{\bar{\alpha}}-\epsilon_{\bar{\beta}}+2 i \gamma} .
$$

As shown in Appendix B, it is possible to separate $F_{i j}$ into two contributions

$$
F_{i j}=\bar{F}_{i j}+\tilde{F}_{i j}
$$


where $\bar{F}_{i j}$ is obtained from the summation over antisymmetric exciton states in Eq. (35), while $\tilde{F}_{i j}$ is given by the contribution of the symmetric exciton states in Eq. (36).

We first consider the symmetric exciton contributions. By using Eqs. (5) and (14) the $\operatorname{sum} \sum_{\bar{m}} v_{\bar{m}}^{(j)} \tilde{\phi}_{\alpha}^{(t)}(\bar{m}) \tilde{\phi}_{\beta}^{\left(t^{\prime}\right)}(\bar{m})$ can be written in terms of wavefunctions of a linear chain:

$$
\sum_{\bar{m}} v_{i}^{(\bar{m})} \tilde{\phi}_{\alpha}^{(t)}(\bar{m}) \tilde{\phi}_{\beta}^{\left(t^{\prime}\right)}(\bar{m})=\sum_{\bar{m}_{i} \in|\bar{i}|} \tilde{\phi}_{\alpha}^{(t)}\left(\bar{m}_{i}\right) \tilde{\phi}_{\beta}^{\left(t^{\prime}\right)}\left(\bar{m}_{i}\right) / N_{i}=\tilde{\psi}_{\alpha}^{(t)}(i) \tilde{\psi}_{\beta}^{\left(t^{\prime}\right)}(i) \frac{1+q_{t} q_{t^{\prime}}+q_{t}^{2} q_{t^{\prime}}^{2}}{3 \sqrt{2^{i-1}}}
$$

where we use the fact that wavefunctions $\tilde{\psi}_{\alpha}^{(t)}(i)$ of different branches for same symmetric exciton state scale to each other as $\left(1, q_{t}, q_{t}^{2}\right)$.

Using Eq. (18), Eq. (35) assumes the form:

$$
\tilde{F}_{i j}(\omega)=\frac{1}{27 \sqrt{2^{i+j-2}}} \sum_{\alpha=1}^{l} \sum_{\beta=1}^{l} \sum_{t=1}^{3} \sum_{t^{\prime}=1}^{3} \frac{\tilde{\psi}_{\alpha}^{(t)}(i) \tilde{\psi}_{\beta}^{\left(t^{\prime}\right)}(i)\left[\tilde{\psi}_{\alpha}^{(t)}(j) \tilde{\psi}_{\beta}^{\left(t^{\prime}\right)}(j)\right]^{*}}{\omega-\epsilon_{\alpha, t}-\epsilon_{\beta, t^{\prime}}+2 i \gamma}\left|1+q_{t} q_{t^{\prime}}+q_{t}^{2} q_{t^{\prime}}^{2}\right|^{2}
$$

As seen from (4) $1+q_{t} q_{t^{\prime}}+q_{t}^{2} q_{t^{\prime}}^{2} \neq 0$, iff $q_{t} q_{t^{\prime}} \equiv 1$, while $\epsilon_{\alpha, 3}=\epsilon_{\alpha, 2}$ (compare Eqs. (A1) and (A2) in [9]). We use these properties in Eq. (19), to finally obtain

$$
\tilde{F}_{i j}(\omega)=\frac{1}{3 \sqrt{2^{i+j-2}}} \sum_{\alpha=1}^{l} \sum_{\beta=1}^{l} \sum_{t=1}^{2} \frac{t \tilde{\psi}_{\alpha}^{(t)}(i) \tilde{\psi}_{\beta}^{(t)}(i)\left[\tilde{\psi}_{\alpha}^{(t)}(j) \tilde{\psi}_{\beta}^{(t)}(j)\right]^{*}}{\omega-\epsilon_{\alpha, t}-\epsilon_{\beta, t}+2 i \gamma} .
$$

We now consider the antisymmetric exciton contribution [Eq. (36)]. To find out which excitons contribute, let us first consider the auxiliary sum $\sum_{s} \sum_{\bar{\alpha}, \bar{\beta}} \sum_{\bar{m}} v_{i}^{(\bar{m})} \phi_{\bar{\alpha}}^{(s)}(\bar{m}) \phi_{\bar{\beta}}^{(s)}(\bar{m})$. Since the vector $v_{i}^{(\bar{m})}$ has non-zero components only for chromophores that belong to the $i$ th generation (chromophores with index $\bar{m}_{i}$ in (14)), only the antisymmetric excitons which start earlier than the $i$-th generation, contribute to the sum. We next calculate the total number of different contributing exciton states. As early discussed, there are $3 \cdot 2^{l-s-1}$ one-exciton states within the exciton of length $s$. Making use of (3) and (14), we obtain

$$
\sum_{s} \sum_{\bar{\alpha}, \bar{\beta}} \sum_{\bar{m}} v_{i}^{(\bar{m})} \phi_{\bar{\alpha}}(\bar{m}) \phi_{\bar{\beta}}(\bar{m})=\sqrt{3 / 2^{i-1}} \sum_{s=1}^{i-1} \sum_{\alpha, \beta=1}^{l-s} 2^{s-1} \psi_{\alpha}^{(l-s)}(i-s) \psi_{\beta}^{(l-s)}(i-s) .
$$

We now turn to the matrix element $\bar{F}_{i j}$ and look for the contribution of exciton states similar to (21). We assume $i \geq j$, but as it will follow from (22), $\bar{F}_{i j}=\bar{F}_{j i}$. Although Eq. (36) contains a product of four wavefunctions (as opposed to two in (21)), it is obvious that the excitons contributing to $\bar{F}_{i j}$ must belong to a generation lower or equal to $i-1$ (otherwise, the two wavefunctions are exactly zero for any $\alpha$ and $\beta$ in (16)). It follows further from the definition (14) of the vector $v_{i}^{(\bar{m})}$ that only the wavefunctions of the $i$-th generation contribute to $\bar{F}_{i j}$. This fixes the length $s$ for the contributing excitons: It must start from the $i$-th generation. $s$ determines the set of allowed values for both quantum numbers $\alpha$ and $\beta$ because both $\phi_{\alpha}^{(s)}(\bar{m})$ and $\phi_{\beta}^{(s)}(\bar{m})$ are present in Eq. (36). Using this argument we obtain $s=s^{\prime}$, resulting in an expression for $\bar{F}_{i j}$, which is very similar to $(21)$ :

$$
\bar{F}_{i j}(\omega)=\frac{1}{\sqrt{2^{i+j-2}}} \sum_{s=1}^{i-1} \sum_{\alpha, \beta=1}^{l-s} \frac{2^{s-1} \psi_{\alpha}^{(l-s)}(i-s) \psi_{\beta}^{(l-s)}(i-s)\left[\psi_{\alpha}^{(l-s)}(j-s) \psi_{\beta}^{(l-s)}(j-s)\right]^{*}}{\omega-\epsilon_{\alpha}^{(l-s)}-\epsilon_{\beta}^{(l-s)}+2 i \gamma}, i \geq j,
$$

where we also used the vector norm (14). 
Both eigenvalues and eigenvectors of the excitons are real (Eqs. (A1) and (A2) in [9]) correspond to the real symmetric tridiagonal matrices.). Using this in Eqs. (20) and (22), we rewrite Eq. (17) as

$$
\begin{array}{r}
F_{j i}(\omega)=F_{i j}(\omega)=\frac{1}{\sqrt{2^{i+j-2}}}\left(\frac{1}{3} \sum_{\alpha=1}^{l} \sum_{\beta=1}^{l} \sum_{t=1}^{2} \frac{t \tilde{\psi}_{\alpha}^{(t)}(i) \tilde{\psi}_{\beta}^{(t)}(i) \tilde{\psi}_{\alpha}^{(t)}(j) \tilde{\psi}_{\beta}^{(t)}(j)}{\omega-\epsilon_{\alpha, t}-\epsilon_{\beta, t}+2 i \gamma}+\right. \\
\left.\sum_{s=1}^{i-1} \sum_{\alpha, \beta=1}^{l-s} \frac{2^{s-1} \psi_{\alpha}^{(l-s)}(i-s) \psi_{\beta}^{(l-s)}(i-s) \psi_{\alpha}^{(l-s)}(j-s) \psi_{\beta}^{(l-s)}(j-s)}{\omega-\epsilon_{\alpha}^{(l-s)}-\epsilon_{\beta}^{(l-s)}+2 i \gamma}\right) .
\end{array}
$$

As can be seen from Eq. (23), the elements of symmetric matrix can be represented as $F_{i j}=\left(1 / \sqrt{2^{i-1}}\right) f_{i j}\left(1 / \sqrt{2^{j-1}}\right)$, with

$$
\begin{array}{r}
f_{j i}(\omega)=f_{i j}(\omega)=\frac{1}{3} \sum_{\alpha=1}^{l} \sum_{\beta=1}^{l} \sum_{t=1}^{2} \frac{t \tilde{\psi}_{\alpha}^{(t)}(i) \tilde{\psi}_{\beta}^{(t)}(i) \tilde{\psi}_{\alpha}^{(t)}(j) \tilde{\psi}_{\beta}^{(t)}(j)}{\omega-\epsilon_{\alpha, t}-\epsilon_{\beta, t}+2 i \gamma}+ \\
\sum_{s=1}^{i-1} \sum_{\alpha, \beta=1}^{l-s} \frac{2^{s-1} \psi_{\alpha}^{(l-s)}(i-s) \psi_{\beta}^{(l-s)}(i-s) \psi_{\alpha}^{(l-s)}(j-s) \psi_{\beta}^{(l-s)}(j-s)}{\omega-\epsilon_{\alpha}^{(l-s)}-\epsilon_{\beta}^{(l-s)}+2 i \gamma} .
\end{array}
$$

In a matrix form, we write

$$
F=A f A, \quad a_{i j}=\delta_{i j} / \sqrt{2^{i-1}} .
$$

The matrix $A$ is diagonal, so that $\left(a^{-1}\right)_{i j}=1 / a_{i j}=\sqrt{2^{i-1}} \delta_{i j}$. The back substitution provides $F^{-1}=A^{-1} f^{-1} A^{-1}$, or

$$
F_{i j}^{-1}=\sqrt{2^{i-1}} f_{i j}^{-1} \sqrt{2^{j-1}} .
$$

The scattering matrix $\bar{\Gamma}_{\bar{m} \bar{n}}(\omega)$ is given in terms of the two-exciton Green's function (8) with matrix elements (26). The relation between the matrix elements of the exciton scattering matrix in the original basis, $\bar{\Gamma}_{\bar{m} \bar{n}}$, and in the basis set of $v_{i}$ [Eq. (14)], $\bar{\Gamma}_{i j}$, is given by an expression, which is similar to Eq. (15):

$$
\bar{\Gamma}_{\bar{m} \bar{n}}=\sum_{i, j=1}^{l} v_{i}^{(m)} \bar{\Gamma}_{i j} v_{j}^{(n) *}
$$

Combining Eqs. (8) and (26), we express the two-exciton scattering matrix in terms of single-exciton wavefunctions and their energies

$$
\bar{\Gamma}_{i j}(\omega)=3 \cdot 2^{i+j-1} \operatorname{Im}\left[f_{i j}^{-1}(\omega)\right] .
$$

Making use of Eqs. (28) in (13), and (27) we obtain the final expression for the nonlinear signal

$$
S\left(\omega_{2}, \omega_{1}\right) \propto-(3 / 2) \mu^{4} \sum_{i, j=1}^{l} 2^{i+j} \operatorname{Im}\left[f_{i j}^{-1}\left(\omega_{1}+\omega_{2}\right)\right],
$$

where the matrix elements $f_{i j}$ are expressed using the one-exciton states [Eq. (24)].

\section{APPENDIX B: EXCITON CONTRIBUTIONS TO THE F MATRIX}

In this section we separate the contribution from the symmetric and antisymmetric excitons to the two-exciton matrix elements $F_{i j}$ and derive Eq. (17). 
We first rewrite Eq. (16) in the following form

$$
F_{i j}(\omega)=\sum_{\bar{m} \bar{n}} v_{i}^{(\bar{m})} v_{j}^{(\bar{n}) *} \sum_{\bar{\beta}} \phi_{\bar{\beta}}(\bar{m}) \phi_{\bar{\beta}}^{*}(\bar{n}) \sum_{\bar{\alpha}} \frac{\phi_{\bar{\alpha}}(\bar{m}) \phi_{\bar{\alpha}}^{*}(\bar{n})}{\omega-\epsilon_{\bar{\alpha}}-\epsilon_{\bar{\beta}}+2 i \gamma} .
$$

The transformation from generalized excitonic states $\bar{\alpha}$ to the symmetric excitons is

$$
\bar{\alpha} \rightarrow(\alpha, t), \epsilon_{\bar{\alpha}} \rightarrow \epsilon_{\alpha, t}
$$

and to the antisymmetric excitons

$$
\bar{\alpha} \rightarrow(\alpha, s), s=1, . ., l-1, \epsilon_{\bar{\alpha}} \rightarrow \epsilon_{\alpha}^{(s)},
$$

where the index $s$ represents the length of an antisymmetric exciton, and $t$ designates one (out of three) types of symmetric excitons. Using Eqs. (31) and (32) in (30), we first obtain

$$
F_{i j}(\omega)=\sum_{\bar{m} \bar{n}} v_{i}^{(\bar{m})} v_{\bar{n}}^{(j) *} \sum_{\bar{\beta}} \phi_{\bar{\beta}}(\bar{m}) \phi_{\bar{\beta}}^{*}(\bar{n})\left[\sum_{t, \alpha} \frac{\tilde{\phi}_{\alpha}^{(t)}(\bar{m}) \tilde{\phi}_{\alpha}^{(t) *}(\bar{n})}{\omega-\epsilon_{\alpha, t}-\epsilon_{\bar{\beta}}+2 i \gamma}+\sum_{s, \alpha} \frac{\phi_{\alpha}^{(s)}(\bar{m}) \phi_{\alpha}^{(s) *}(\bar{n})}{\omega-\epsilon_{\alpha}^{(s)}-\epsilon_{\bar{\beta}}+2 i \gamma}\right] .
$$

Applying rules (31) and (32) in Eq. (33) for states with $\bar{\beta}$, we rewrite Eq. (16) as

$$
F_{i j}=\bar{F}_{i j}+\tilde{F}_{i j}+2 \xi_{i j}
$$

In Eq. (34), $\bar{F}_{i j}$ is given by the antisymmetric excitons

$$
\bar{F}_{i j}=\sum_{s, s^{\prime}=1}^{l-1} \sum_{\alpha=1}^{s} \sum_{\beta=1}^{s^{\prime}} \frac{\sum_{\bar{m}} v_{i}^{(\bar{m})} \phi_{\alpha}^{(s)}(\bar{m}) \phi_{\beta}^{\left(s^{\prime}\right)}(\bar{m}) \sum_{\bar{n}}\left[v_{\bar{n}}^{(j)} \phi_{\alpha}^{(s)}(\bar{n}) \phi_{\beta}^{\left(s^{\prime}\right)}(\bar{n})\right]^{*}}{\omega-\epsilon_{\alpha}^{(s)}-\epsilon_{\beta}^{(s \prime)}+2 i \gamma},
$$

while the contribution of the symmetric excitons to the two-exciton Green's function is

$$
\tilde{F}_{i j}=\sum_{t=1}^{3} \sum_{t^{\prime}=1}^{3} \sum_{\alpha=1}^{l} \sum_{\beta=1}^{l} \frac{\sum_{\bar{m}} v_{i}^{(\bar{m})} \tilde{\phi}_{\alpha}^{(t)}(\bar{m}) \phi_{\beta}^{\left(t^{\prime}\right)}(\bar{m}) \sum_{\bar{n}}\left[v_{\bar{n}}^{(j)} \tilde{\phi}_{\alpha}^{(t)}(\bar{n}) \tilde{\phi}_{\beta}^{\left(t^{\prime}\right)}(\bar{n})\right]^{*}}{\omega-\epsilon_{\alpha, t}-\epsilon_{\beta, t^{\prime}}+2 i \gamma} .
$$

The cross term formed by combination of symmetric and antisymmetric excitons,

$$
\xi_{i j}=\sum_{t, \alpha} \sum_{s, \beta} \frac{\sum_{\bar{m}} v_{i}^{(\bar{m})} \tilde{\phi}_{\alpha}^{(t)}(\bar{m}) \phi_{\beta}^{(s)}(\bar{m}) \sum_{\bar{n}}\left[v_{\bar{n}}^{(j)} \tilde{\phi}_{\alpha}^{(t)}(\bar{n}) \phi_{\beta}^{(s)}(\bar{n})\right]^{*}}{\omega-\epsilon_{\alpha, t}-\epsilon_{\beta}^{(s)}+2 i \gamma}
$$

vanishes. To prove that, we consider the sum

$$
\sum_{\bar{m}} v_{i}^{(\bar{m})} \tilde{\phi}_{\alpha}^{(t)}(\bar{m}) \phi_{\beta}^{(s)}(\bar{m})=\sum_{\bar{m}_{i}=1}^{N_{i}} \tilde{\phi}_{\alpha}^{(t)}\left(\bar{m}_{i}\right) \phi_{\beta}^{(s)}\left(\bar{m}_{i}\right) / \sqrt{N_{i}} \quad \forall i, t, s .
$$

We break the summation (38) as $\sum_{\bar{m}_{i}=1}^{N_{i}}=\sum_{\bar{m}_{i}=1}^{N_{i} / 2}+\sum_{\bar{m}_{i}=N_{i} / 2+1}^{N_{i}}$. As seen from Fig. 3, the antisymmetric wavefunctions change sign upon rotation with respect to rotation the exciton origin $A_{\bar{m}} \phi_{\beta}^{(s)}\left(\bar{m}_{i}\right)=-\phi_{\beta}^{(s)}\left(\bar{m}_{i}+N_{i} / 2\right)$, for any chromophore that belongs to the left semiplane formed by dividing the exciton plane by $A_{\bar{m}}$ axis, $\bar{m}_{i} \leq N_{i} / 2$. At the same time, the symmetric wavefunctions do not change sign under such an operation, and $A_{\bar{m}} \tilde{\phi}_{\beta}^{(t)}\left(\bar{m}_{i}\right)=$ $\tilde{\phi}_{\beta}^{(t)}\left(\bar{m}_{i}+N_{i} / 2\right)$. Therefore, $\sum_{\bar{m}_{i}=1}^{N_{i} / 2}=-\sum_{\bar{m}_{i}=N_{i} / 2+1}^{N_{i}}$, the sum (38) vanishes, and Eqs. (34) and (16) are equivalent. 


\section{References}

[1] M.R. Shortreed, S.F. Swallen, Z.-Y. Shi, W. Tan, Z. Xu, J.S. Moore, R. Kopelman, J. Phys. Chem. B 101, 6318 (1997).

[2] R. Kopelman, M.R. Shortreed, Z.-Y. Shi, W. Tan, Z. Xu, C. Devadoss, J.S. Moore, A. Bar-Haim, Y. Klafter, Phys. Rev. Lett. 78, 1239 (1997).

[3] M. Shortreed, Z.-Y. Shi, R. Kopelman, Mol. Cryst. Liq. Crist. 283, 95 (1996).

[4] D.A. Tomalia, A.M. Naylor, W.A. Goddard, Angew. Chem. Int. Ed. Engl. 29, 138 (1990).

[5] M.K. Lothian-Tomalia, D.M. Hedstrand, D.A. Tomalia, A.B. Padias, H.K.Hall, Jr., Tetrahedon 53, 15495 (1997).

[6] V. Balzani, S. Campagna, G. Denti, A. Juris, S. Serroni, M. Venturi, Acc. of Chem. Res. 31, 26 (1998).

[7] M. Venturi, S. Serroni, A. Juris, S. Campagna, V. Balzani, Topics in Current Chemistry, 197 (Springer, Berlin, 1998).

[8] J. Freéchet, Science 263, 1710 (1994).

[9] E.Y. Poliakov, V. Chernyak, S. Tretiak, S. Mukamel, J. Chem. Phys. (in press).

[10] Z. Xu, M. Kahr, K.L. Walker, C.L. Wilkins, J.S. Moore, J. Am. Chem. Soc. 116, 4537 (1994).

[11] C. Devadoss, P. Bharathi, J.S. Moore, J. Am. Chem. Soc. 118, 9635 (1996).

[12] D.R. Kanis, M.A. Ratner, T.J. Marks, Chem. Rev. 94, 195 (1994).

[13] J.L.Brédas, C. Adant, P. Tackyx, A. Persoons, B.M. Pierce, Chem. Rev. 94, 243 (1994).

[14] A.S. Davydov, Theory of Molecular Excitons, (Plenum, New York, 1971).

[15] In Excitons, edited by E.I. Rashba, M.D. Sturge (North-Holland, Amsterdam, 1982); V.B. Broude, E.I. Rashba, E.F. Sheka, Spectroscopy of Molecular Excitons (Springer, Berlin, 1985).

[16] S. Tretiak, V. Chernyak, S. Mukamel, J. Phys. Chem. B, 102, 3310 (1998).

[17] S. Mukamel, Principles of Nonlinear Optical Spectroscopy (University Press, Oxford, 1995).

[18] V. Chernyak, S. Mukamel, Phys. Rev. B 48, 2470 (1993); J. Chem. Phys. 100, 2974 (1994).

[19] In J-aggregates, edited by T. Kobayaschi (World Scientific, Singapore, 1996).

[20] V. Chernyak, N. Wang, S. Mukamel, Phys. Rep. 263, 213 (1995). 